\title{
Spontaneous Extracranial Vertebral Artery Dissociation: A Case Report and Review of the Literature
}

\author{
D. Kamakura, Y. Iida, K. Hasegawa, S. Tsuge, K. Fukutake, K. Nakamura, H. Takahashi, A. Wada \\ Department of Orthopedic Surgery, Toho University School of Medicine, Tokyo, Japan \\ Email: nyagira@med.toho-u.ac.jp
}

How to cite this paper: Kamakura, D., Iida, Y., Hasegawa, K., Tsuge, S., Fukutake, K., Nakamura, K., Takahashi, H. and Wada, A. (2018) Spontaneous Extracranial Vertebral Artery Dissociation: A Case Report and Review of the Literature. Open Journal of Orthopedics, 8, 221-226.

https://doi.org/10.4236/ojo.2018.86025

Received: April 29, 2018

Accepted: June 1, 2018

Published: June 4, 2018

Copyright $\odot 2018$ by authors and Scientific Research Publishing Inc. This work is licensed under the Creative Commons Attribution International License (CC BY 4.0).

http://creativecommons.org/licenses/by/4.0/

\begin{abstract}
We encountered a 36-year-old female who developed extracranial vertebral artery dissociation (EVAD), which is a rare condition in Japan. The disease developed with sudden severe right side neck pain and right upper arm pain. No abnormality was observed in the spinal canal, intervertebral discs, spinal cord, or nerve roots on MRI of the cervical spine, but dilation and double lumen were noted in the vertebral artery (VA) at the C4/5 level. The presence of aneurysm was confirmed by VA angiography, and EVAD was diagnosed. The symptoms were resolved by conservative treatment of pain, and the aneurysm shrank and disappeared with time on VA angiography. Even though neck and upper limb pain are the only clinical symptom and there is no brain dysfunction, such as disturbance of consciousness and vertigo, when symptoms develop acutely, this pathology should be included in differential diagnosis and orthopedists should carefully observe the vertebral artery, as well as the spinal canal, in evaluating cervical MRI.
\end{abstract}

\section{Keywords}

Extracranial Vertebral Artery Dissection, Neck Pain, Cervical Spine

\section{Introduction}

Spontaneous dissection of the vertebral artery (VAD) is increasingly recognized as an important cause of stroke in young adults. VAD is classified according to the location of dissection as intracranial VAD (IVAD) and extracranial VAD (EVAD), and EVAD is a rare disorder in Japan. EVAD is often accompanied by sudden onset of headache, nausea, and vertigo, in addition to neck pain, following a recent neck injury, including minor trauma such as chiropractic therapy. 
Anticoagulant therapy is effective for managing this disorder, but prompt diagnosis is essential to obtain a favorable outcome. We encountered a patient who visited our department for a chief complaint of severe neck pain and was diagnosed with EVAD, and in whom the disease was remitted by conservative therapy. We report the case with a literature review.

\section{Case Report}

Severe pain suddenly developed in the right side of the neck over the scapular region and right upper arm without trauma in a 36-year-old woman. She visited an orthopedic clinic and was diagnosed with cervical disc herniation. Medication with a non-steroidal anti-inflammatory drug (NSAID) was started, but pain was not relieved. She was referred to our hospital for close examination 4 days after onset. She had no particular past or familial medical history. The first examination at our hospital showed clear consciousness; body temperature, $36.7^{\circ} \mathrm{C}$; blood pressure, 132/72 mmHg; heart rate, 66/min; vertigo, (-); nausea, (-). The range of motion of the neck region was strongly limited due to intractable pain, Jackson and Spurling tests were both negative, manual muscle tests were normal in both upper and lower extremities, no sensory disturbance was noted, and all deep tendon reflexes were normal.

Cervical disc herniation and cervical spondylosis were suspected based on the clinical course and symptoms, and the cervical spine was examined by imaging. Plain radiography of the cervical spine was normal. On cervical MRI, no space-occupying lesion was present in the spinal canal and nerve root foramen, but dilation of the lumen of the right vertebral artery (VA) and double lumen were noted at the $\mathrm{C} 4$ intervertebral disc level in the horizontal section on T2-weighted imaging (Figure 1). VA angiography indicated a 2-mm aneurysm at the same level on 3D-CT (Figure 2), and EVAD was diagnosed.

Conservative therapy by local fixation with a cervical collar, an oral NSAID, and blood pressure control were initiated, and neck pain gradually remitted. VA angiography aimed at endovascular treatment was performed 3 weeks after admission, and showed shrinkage of the aneurysm. Therefore, no endovascular or surgical treatment was performed and the patient was discharged. On VA angiography performed after 3 months, the aneurysm had vanished (Figure 3). As of 2 years after onset, no neck pain has recurred and the patient had returned to her previous work.

\section{Discussion}

For a female patient in her $30 \mathrm{~s}$ who visits an orthopedist for chief complaints of neck, scapular or upper limb pain without traumatic history, cervical spondylodiscitis and neoplasm are differentiated with reference to medical history, in addition to differential diagnosis of cervical disc herniation, cervical spondylosis, and thoracic outlet syndrome during the course of diagnosis. EVAD is often accompanied by stroke, nausea, and vertigo, in addition to neck pain, and the 

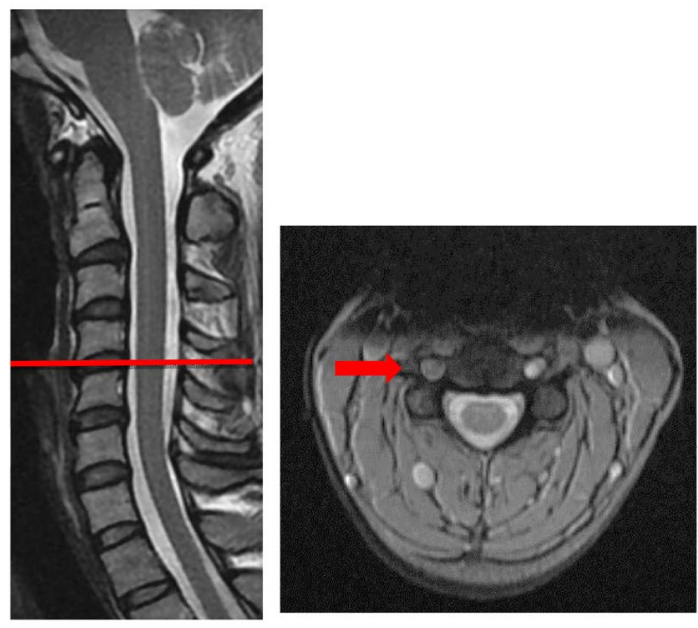

Figure 1. Head-and-neck MRI on admission. There was no space-occupying lesion in the spinal canal, but dilation of the lumen and double lumen were noted in the right vertebral artery at the C4/5 disc level in the horizontal section on T2-weighted imaging (red arrow). The red line on the sagittal image (left) indicates the level of the axial slice (right).

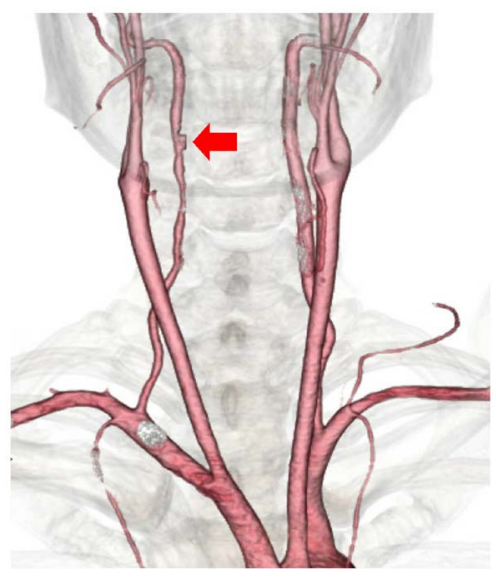

Figure 2. Head-and-neck 3D-CT angiography showed an aneurysm of $2 \mathrm{~mm}$ in the right vertebral artery at the C4/5 disclevel (red arrow).
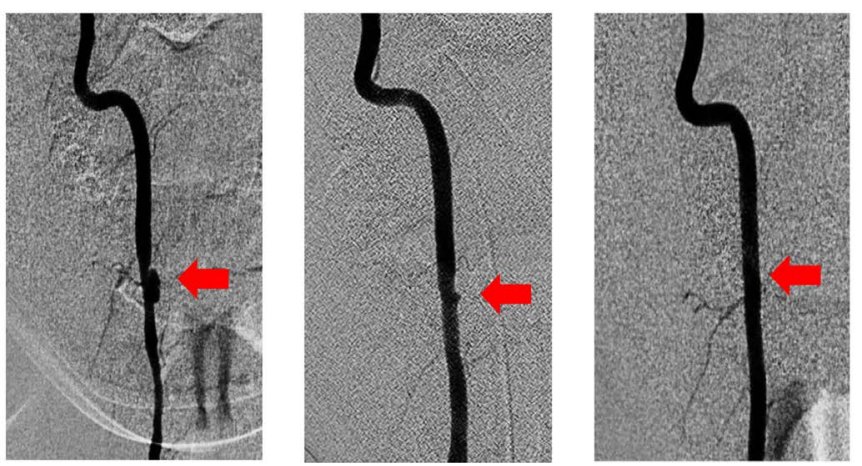

Figure 3. Time-course changes after admission. VA angiography was performed three days after admission (left). With the aim of endovascular therapy, VA angiography was again performed three weeks after admission. The aneurysm had shrunk, and endovascular therapy was cancelled (center). The aneurysm subsequently disappeared 3 months after admission (right). Red arrows indicate the position of the aneurysm. 
disease is likely to be overlooked when these accompanying symptoms are absent [1].

In Japan, $232(82 \%)$ and $30(11 \%)$ of 283 cases diagnosed as VAD were IVAD and EVAD, respectively [2], whereas $378(74 \%)$ and $134(26 \%)$ of 512 cases were IVAD and EVAD, respectively, in a systematic review of cases in western countries [3]. These data demonstrate that the frequency of EVAD in Japan is less than half that in western countries. The mean onset age of EVAD was younger than that of IVAD, and the cause was often trauma, including in mild cases. The cause of stroke was SAH in $43 \%$ and ischemic stroke (IS) in 57\% of IVAD cases, whereas IS accounted for $97 \%$ of EVAD cases [1] [2] [3].

The histology of the VA is markedly different in the epidural and intradural regions [4]. The intradural artery is thin and contains fewer elastic fibers, in which dissection readily occurs, and the external elastic lamina is absent and the adventitial collagen fiber layer is thin, which allows ready formation of an aneurysm. For these reasons, IVAD may more readily induce SAH compared with EVAD.

Pathologically, there are two developmental patterns of VAD: a dissecting aneurysm type, in which a false lumen dilates outward and forms an aneurysm causing subarachnoid hemorrhage (SAH); and an aneurysm and dissociative stenosis type, in which a false lumen dilates inward and narrows the vascular lumen, causing IS (Figure 4).

The main mechanism of cerebral ischemia in EVAD is considered to be embolism caused by formation of a thrombus in the dissected region, but aneurysm formation is observed on imaging in many cases of SAH. Accordingly, although anticoagulant therapy (heparin and subsequent warfarin administration) from the acute phase is recommended for treatment of EVAD [5] [6], antithrombotic therapy is contraindicated when aneurysm formation is clearly observed because of concern regarding aggravation of SAH. The dissected region becomes patent after 3 - 6 months, even though the region is completely obstructed in the acute

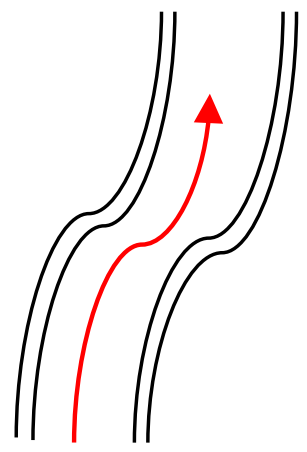

Normal

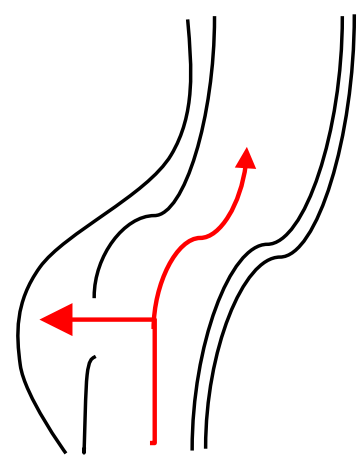

(a)

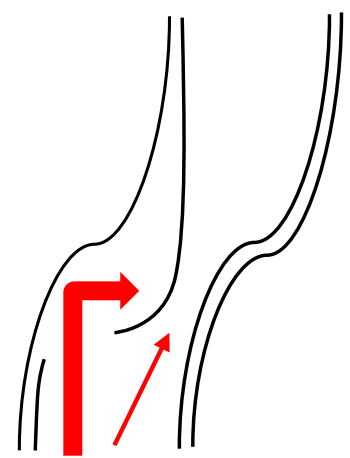

(b)

Figure 4. Classification of extracranial vertebral artery dissociation. (a) Dissecting aneurysm type; (b) Aneurysm and dissociative stenos is type. Red arrows show the blood flow of the VA. 
phase in $60 \%$ - $80 \%$ of EVAD cases [7]. After this phase, the risks of recurrent cerebral infarction and dissociation are low, and the outcome is favorable in many patients [8]. Therefore, prevention of recurrence by continuation of antithrombotic therapy limited to 3 - 6 months after onset is recommended [5], but there is no evidence for its efficacy. In our patient, the lesion was a relatively localized aneurysm and dissociative stenos are type. Fortunately, no brain symptom developed throughout the course and the symptoms were remitted by treatment of neck pain alone. Antithrombotic therapy was not performed because shrinkage and disappearance of the aneurysm was confirmed over the course by VA angiography. To the best of our knowledge, there is no systematic report on the correlation between the size of aneurysm and spontaneous shrinkage for EVAD, but it is likely that many small aneurysm cases might have overlooked or misdiagnosed in Japan.

The extracranial VA ascends toward the cranial side through the transverse processes from the $\mathrm{C} 6$ vertebrae, and contacts the nerve roots in the transverse processes. There are several reports of radiculopathy caused by compression of VA dissociation-associated vascular dilatation or aneurysm [9]-[14]. Silbert et al. described a case of a previously healthy 43-year-old woman who presented with neck pain and left arm weakness, in which MRI and MR angiography revealed compression of the C5 and C6 nerve roots by expansion of the vertebral artery wall within the intervertebral foramina associated with vertebral artery dissection [9]. Our patient complained of neck pain over the right scapular region and upper arm, but nerve root compression could not be identified on MRI because the aneurysm was small. However, we noticed laterality of the VA in the horizontal section on cervical MRI, although we are orthopedists who mainly diagnose and treat the spine, spinal cord, and peripheral nerves in patients with pain and paralysis. We suspected double lumen of the right vertebral artery and we were able to diagnose EVAD on VA angiography. This may largelybe due to improvement of the image quality of MRI, but it may also have been because we recognized the importance of careful observation of the entire image, including vascular lesions, rather than just the spine, spinal cord, intervertebral discs, and nerve roots.

\section{Conclusion}

In conclusion, we encountered a 36-year-old female patient in whom EVAD developed without a history of trauma. This case demonstrates that EVAD should be considered as a cause in middle-aged patients who complain of acute severe neck pain, even if this is not accompanied by stroke or symptoms of cranial neurological disorder such as nausea and vertigo.

\section{Consent}

The patient gave informed consent to submit this manuscript for publication.

\section{Competing Interests}

The authors declare that there is nothing to disclose regarding the publication of 
this manuscript.

\section{References}

[1] Kobayashi, H., Morishita, T., Ogata, T., Matsumoto, J., Okawa, M., Higashi, T., et al. (2016) Extracranial and Intracranial Vertebral Artery Dissections: A Comparison of Clinical Findings. Journal of Neurological Sciences, 362, 244-250. https://doi.org/10.1016/j.jns.2016.01.062

[2] Takagi, M. (2002) Cryptogenic Stroke. Brain and Nerve, 54, 203-211. (In Japanese)

[3] Gottesman, R.F., Sharma, P., Robinson, K.A., Arnan, M., Tsui, M., Ladha, K., et al. (2012) Clinical Characteristics of Symptomatic Vertebral Artery Dissection. A Systematic Review. Neurologist, 18, 245-254. https://doi.org/10.1097/NRL.0b013e31826754e1

[4] Wilkinson, I.M. (1972) The Vertebral Artery: Extracranial and Intracranial Structure. Arch Neurol, 27, 392-396.

https://doi.org/10.1001/archneur.1972.00490170024004

[5] Schievink, W.I. (2001) Spontaneous Dissection of the Carotid and Vertebral Arteries. New England Journal of Medicine, 344, 898-906.

https://doi.org/10.1056/NEJM200103223441206

[6] Bassi, P., Lattuada, P. and Gomitoni, A. (2003) Cervical Cerebral Artery Dissection: A Multicenter Prospective Study (Preliminary Report). Neurological Sciences, 24, S4-S7. https://doi.org/10.1007/s100720300025

[7] Engelter, S.T., Brandt, T., Debette, S., Caso, V., Lichy, C., Pezzini, A., et al. (2007) Antiplatelets versus Anticoagulation in Cervical Artery Dissection. Stroke, 38, 2605-2611. https://doi.org/10.1161/STROKEAHA.107.489666

[8] Sacco, R.L., Adams, R., Albers, G., Alberts, M.J., Benavente, O., Furie, K., et al. (2006) Guidelines for Prevention of Stroke in Patients with Ischemic Stroke or Transient Ischemic Attack: A Statement for Healthcare Professionals from the American Heart Association/American Stroke Association Council on Stroke: Co-Sponsored by the Council on Cardiovascular Radiology and Intervention: The American Academy of Neurology Affirms the Value of This Guideline. Circulation, 113, e409-e449. https://doi.org/10.1161/01.STR.0000199147.30016.74

[9] Silbert, B.I. (2013) Vertebral Artery Dissection as a Cause of Cervical Radiculopathy. Asian Spine, 7, 335-338. https://doi.org/10.4184/asj.2013.7.4.335

[10] Berroir, S., Sarazin, M. and Amarenco, P. (2002) Vertebral Artery Dissection Presenting as Neuralgic Amyotrophy. Journal of Neurology Neurosurgery \& Psychiatry, 72, 552-553.

[11] Crum, B., Mokri, B. and Fulgham, J. (2000) Spinal Manifestations of Vertebral Artery Dissection. Neurology, 55, 304-306. https://doi.org/10.1212/WNL.55.2.304

[12] McGillion, S.F., Weston-Simons, S. and Harvey, J.R. (2009) Vertebral Artery Dissection Presenting with Multilevel Combined Sensorimotor Radiculopathy: A Case Report and Literature Review. Journal of Spinal Disorders \& Techniques, 22, 456-458. https://doi.org/10.1097/BSD.0b013e318185d31a

[13] Uemura, H., Kuroda, S., Ushikoshi, S., Seki, T., Ishikawa, T., Hida, K., et al. (2004) Complete Resolution of Radiculopathy Due to Cervical Vertebral Artery Dissection after Intravascular Treatment: A Case Report. Neurological Surgery, 32, 361-365.

[14] Hamaguchi, H. (2012) A Case of Vertebral Artery Dissection Diagnosed with Muscle Weakness of the Right Upper Extremity. The Journal of Japanese College of Angiology, 52, 137-140. 